\title{
Herramientas que facilitan el aprendizaje colaborativo en entornos virtuales: nuevas oportunidades para el desarrollo de las ecologías digitales de aprendizaje
}

\section{Tools that facilitate collaborative learning in virtual environments: new opportunities for the development of digital learning ecologies}

\author{
Núria HernándeZ-Sellés ${ }^{1}$ \\ nuria@lasallecampus.es \\ La Salle Campus Madrid, España
}

\section{Resumen:}

Esta aportación deriva de un proyecto de investigación en el que se analiza una propuesta pedagógica y tecnológica para el diseño de procesos de aprendizaje CSCL (Computer Supported Collaborative Learning). En este trabajo se presenta un análisis de las herramientas en el aprendizaje colaborativo a partir de la percepción del alumnado participante en el estudio. Se articula sobre un análisis de las principales soluciones tecnológicas aplicadas en el campo del CSCL, distinguiendo entre el uso colaborativo de la tecnología y la tecnología colaborativa. Se presentan los resultados de un estudio cuantitativo ex post facto de carácter no experimental basado en el método de encuesta en el que participan 106 estudiantes de cinco asignaturas pertenecientes a los grados de Maestro Educación Primaria e Infan-

\begin{abstract}
:
This contribution derives from a research project that analyzes a pedagogical and technological proposal for the design of CSCL (Computer Supported Collaborative Learning) learning processes. This work presents an analysis of the tools in collaborative learning based on the perception of the students participating in the study. It is based on an analysis of the main technological solutions applied in the field of CSCL, distinguishing between the collaborative use of technology and collaborative technology. The research entails an ex post facto non-experimental quantitative study based on the survey method conducted with 106 students of five subjects belonging to the degrees of Primary and Infant Education at Campus La Salle Madrid. Students developed project-based learning with a CSCL-based methodology; analy-
\end{abstract}

1 Dirección para correspondencia (correspondence address):

Núria Hernández-Sellés. Centro Superior de Estudios Universitarios La Salle. Facultad de Educación y Ciencias Sociales, C/La Salle, 10, 28023, Madrid (España) 
Herramientas que facilitan el aprendizaje colaborativo en entornos virtuales: nuevas oportunidades para el desarrollo de las ecologías digitales de aprendizaje

NúRIA HeRnándEZ-SELLÉS

til del Campus La Salle en Madrid y que desarrollaron aprendizaje basado en proyectos con una metodología basada en CSCL; se presentan análisis atendiendo a la variable edad de los estudiantes y los años de experiencia trabajando en entornos virtuales. Los estudiantes valoran que las tecnologías más útiles en el CSCL son la videoconferencia y la mensajería instantánea, como WhatsApp, frente a otras herramientas como Wikis, Blogs o Redes Sociales. Al margen de la situación derivada de la pandemia del COVID-19, los sistemas de videoconferencia y mensajería instantánea desde dispositivos móviles no han sido las herramientas integradas de forma habitual en las plataformas virtuales o incorporadas en los procesos CSCL, sin embargo, los estudiantes valoran positivamente su utilidad en los procesos de colaboración. En este sentido, las instituciones de educación superior deberían promover una amplia reflexión en torno a las herramientas que favorecen los procesos de interacción y los aprendizajes en colaboración, aprovechando el gran esfuerzo que se ha tenido que asumir en la situación de pandemia, que se ha sostenido en gran medida gracias a los ecosistemas digitales.

\section{Palabras clave:}

Aprendizaje cooperativo/colaborativo; Educación superior; Estrategias de aprendizaje; Comunicación mediada por tecnologías; ecologías de aprendizaje. sis are presented according to the age of the students and the years of experience working in virtual environments. Students value that the most useful technologies in CSCL are videoconferencing and instant messaging, such as WhatsApp, compared to other tools such as Wikis, Blogs or Social Networks. Aside from the situation derived from the COVID-19 pandemic, videoconferencing and instant messaging systems do not tend to be integrated into virtual platforms or incorporated into CSCL. However, students positively value their usefulness in collaborative processes. In this sense, higher education institutions should promote extensive reflection on the tools that favor interaction processes and collaborative learning, taking advantage of the great effort that has been made during the pandemic situation, which has largely been sustained by digital ecosystems.

\section{Keywords:}

Cooperative / collaborative learning; Higher education; Learning strategies; Communication mediated by technologies; Learning ecologies.

\section{Résumé:}

Cette contribution découle d'un projet de recherche dans lequel est analysée une proposition pédagogique et technologique pour la conception de processus d'apprentissage CSCL (Computer Supported Collaborative Learning). Ce travail présente une analyse des outils d'apprentissage collaboratif basée sur la perception des étudiants participant à l'étude. Il s'articule sur une analyse des principales solutions technologiques appliquées dans le domaine du CSCL, en distinguant I'utilisation collaborative de la technologie et la technologie collaborative. Les résultats d'une étude quantitative ex post facto de caractère non expérimental basée sur la méthode de l'enquête dans laquelle 106 étudiants de cinq matières appartenant aux diplômes d'éducation primaire et de la petite enfance au Campus de La Salle à Madrid et qui ont développé I'apprentissage par projet avec une méthodologie basée sur CSCL sont présentés; les analyses sont présentées en fonction de la variable âge des étudiants et des années d'expérience de travail dans des environnements virtuels. Les étudiants estiment que les technologies les plus utiles en matière d'EACL sont la vidéoconférence et la messagerie instantanée, telle que WhatsApp, par rapport à d'autres outils tels que les wikis, les blogs ou les réseaux sociaux. En dehors de la situation découlant de la pandémie COVID-19, les systèmes de vidéocon- 
férence et de messagerie instantanée à partir d'appareils mobiles n'ont pas été les outils habituellement intégrés dans les plateformes virtuelles ou incorporés dans les processus $\mathrm{CSCL}$, cependant, les étudiants apprécient positivement leur utilité dans les processus de collaboration. En ce sens, les établissements d'enseignement supérieur doivent promouvoir une large réflexion autour des outils qui favorisent les processus d'interaction et l'apprentissage collaboratif, en profitant du grand effort qu'il a fallu assumer dans la situation de pandémie, qui a été soutenu en grande partie grâce aux écosystèmes numériques.

\section{Mots-clés:}

Apprentissage coopératif/collaboratif; enseignement supérieur; stratégies d'apprentissage; communication médiée par la technologie; écologies d'apprentissage.

Fecha de recepción: 29-01-2021

Fecha de aceptación: 24-03-2021

\section{Introducción}

Cuando un sistema social se ve perturbado, del modo en que el nuestro lo ha sido como consecuencia del COVID-19, emerge un movimiento de supervivencia por parte de todos sus elementos que se articula en un enorme esfuerzo creativo en la búsqueda de la necesaria adaptación. Nuestras ecologías de aprendizaje han vivido un punto de inflexión en el contexto de pandemia que, en el ámbito educativo, nos ha hecho adoptar globalmente modelos de trabajo y aprendizaje flexibles mediados por las tecnologías, incluso en los ámbitos más vulnerables (DarlingHammond y Hyler, 2020).

De este modo, los contextos formales, informales y no formales que componen nuestras ecologías de aprendizaje se han concentrado, durante este periodo, en el escenario virtual, omitiendo de manera forzada los entornos presenciales, al producirse en una migración abrupta que se ha esforzado por integrar los sistemas tecnológicos con los culturales y sociales (González-Sanmamed, Sangrà, Estévez y Souto, 2020; González-Sanmamed, Muñoz-Carril y Santos, 2019).

Es por ello que ahora más que nunca es necesario preservar esta mirada integradora que contempla los aspectos tecnológicos junto con los pedagógicos y sociales de los aprendizajes (Garrison, Cleveland-Innes y Fung, 2010; Onrubia y Engel, 2012, Hernández, González y Muñoz, 2014a). A menudo se obvia la articulación (y con ello la mirada) de la interacción social, y los estudios nos muestran que esta no ocurre de forma espontánea en los contextos virtuales de aprendizaje, ni tampoco en los profesionales. Sin esta interacción no es posible sostener el desarrollo cognitivo y, cuan- 
Herramientas que facilitan el aprendizaje colaborativo en entornos virtuales: nuevas oportunidades para el desarrollo de las ecologías digitales de aprendizaje Núria HeRnández-SeLlés

do se da por sentado, lo más probable es que los grupos se socialicen en un nivel muy bajo, lo que lleva a sentimientos individuales de aislamiento y, en definitiva, a una mala experiencia de aprendizaje (Kwon, Liu y Johnson, 2014; Reyes, Brackett, Rivers, White y Salovey, 2012).

\section{Marco Teórico}

El Computer Supported Collaborative Learning (CSCL) ha cobrado gran relevancia en los contextos de educación en línea porque, precisamente, sostiene las dimensiones que se han apuntado anteriormente: integra los sistemas tecnológicos con los culturales o cognitivos y los sociales. De este modo, la colaboración bien estructurada consigue promover el aprendizaje a través de procesos socio-cognitivos de negociación basados en la presencia social y emocional, que sustenta el aprendizaje mediado por tecnologías, mejorando los resultados de aprendizaje individual e incrementando la satisfacción de los estudiantes (Johnson, Johnson y Stanne, 2000; Oakley, Felder, Brent y Elhajj, 2004; King, 2007; Medina y Suthers, 2008; Kwon, et al., 2014). En el CSCL el aprendizaje se desarrolla a través de procesos complejos que conllevan: a) la definición de un objetivo común, que constituirá un desafío para los grupos e implicará el desarrollo de los aprendizajes/competencias; b) la organización del grupo para dar respuesta al desafío; c) la cooperación y negociación para ofrecer la solución; y d) la co-evaluación o evaluación por pares, tanto del proceso como del resultado del trabajo en grupo (Scardamalia y Bereiter, 1994; Johnson y Johnson 2004).

Las tendencias relativas a la investigación en torno al CSCL, en concreto aquellas centradas en identificar los factores que influyen en CSCL, se relacionan fundamentalmente con tres dimensiones, estando dos de ellas vinculadas al ámbito tecnológico: 1) desarrollo del pensamiento crítico y la argumentación; 2) diseño de entornos virtuales y 3) uso de herramientas para guiar los aprendizajes (Tang, Tsai y Lin, 2014).

Como se va deduciendo, en el CSCL los roles de los docentes se relacionan con el de experto en la materia o contenido curricular; con un rol gestor de las tecnologías; con el de diseñador de experiencias que articulen la presencia cognitiva y social; con el rol facilitador y guía de las experiencias, durante el proceso de interacción de los grupos; y con el evaluador, o diseñador de e-evaluaciones complejas que comprometan 
a los estudiantes y los involucren en el proceso de aprendizaje (Garrison \& Clevaland-Innes, 2005; Abdu, De Groot, \& Drachman, 2012; Hernández, Muñoz y González, 2018).

En esta diversidad de roles, la elección del medio o ecosistema de medios resulta clave para sostener la socialización en la educación en línea, ya que debe contemplar la estructuración de los intercambios profesor-alumno y alumno-alumno (de forma sincrónica o asincrónica) y facilitar la observación por parte del docente de los procesos grupales, es decir, sustentar la comunicación, la colaboración y la coordinación (Strijbos et al. 2004).

Los estudios señalan que las herramientas de colaboración en línea tienen una influencia positiva y significativa en la interacción de los estudiantes en sus grupos de trabajo y en el apoyo emocional intragrupal (Bowman \& Akcaoglu, 2014; Hamid, Waycott, Kurnia, \& Chang, 2015, Hernández-Sellés, Muñoz-Carril, González-Sanmamed, 2019). Estas conexiones son importantes, dado que el apoyo emocional intragrupal tiene una influencia positiva y significativa en el aprendizaje colaborativo (Kwon et al., 2014; Hernández-Sellés, Muñoz-Carril, GonzálezSanmamed, 2019). Por otro lado, se deben seleccionar herramientas colaborativas para sostener la resolución de problemas, integrando los recursos sociales que faciliten el aprendizaje (Lu, Lajoie, \& Wiseman, 2010; Pachler, Daly, Mor y Mellaral, 2010).

Strijbos, Martens y Jochems (2004) presentaron una distinción interesante respecto a la tecnología colaborativa y al uso colaborativo de la tecnología, refiriéndose la primera a las herramientas diseñadas y destinadas para la colaboración y la segunda a cualquier medio-tecnología que pueda sostener la colaboración y el aprendizaje grupal (comunicación, coordinación y colaboración).

Siguiendo esta clasificación, podemos agrupar herramientas como Trello, Mural, Voice Thread, Microsoft Teams, Google Drive para identificarlas como tecnología colaborativa, ya que se han diseñado estructuralmente con el fin de favorecer la colaboración a través de escenarios y herramientas de visualización y argumentación. Lu, et al. (2010) clasifican la tecnología colaborativa en dos tipologías: por un lado, las herramientas de visualización y por otro las de argumentación. Las primeras generan oportunidades para construir representaciones visuales de la información, cara a mejorar el proceso de análisis por parte del grupo. Las segundas, mejoran la experiencia de intercambio de los comentarios, 
Herramientas que facilitan el aprendizaje colaborativo en entornos virtuales: nuevas oportunidades para el desarrollo de las ecologías digitales de aprendizaje Núria HeRnández-SeLlés

argumentaciones y discusiones, a través de herramientas de representación como notas, fichas, comentarios vinculados a un producto, etc. En esta misma línea, las herramientas Spiral y Peergrade facilitan la evaluación por pares, contemplando tanto la evaluación formativa como la sumativa y facilitando la colaboración de los estudiantes.

Por otra parte, estarían las tecnologías que pueden sostener la colaboración y el aprendizaje grupal; así como la comunicación, colaboración y coordinación, según identifican Strijbos, et al. (2004). La mayoría de los LMS (Learning Management System) o EVA (Entorno Virtual de Aprendizaje) que se utilizan en las instituciones de educación superior incorporan una diversidad de herramientas para la colaboración, tales como foros, video-foros, blogs, wikis o chats, para la colaboración (sincrónica o asincrónica). Otras herramientas que facilitan la generación compartida son: herramientas de marcadores sociales como Diigo, que permite a los estudiantes recopilar, anotar, organizar y compartir recursos en línea; o herramientas de colaboración multimedia como Padlet, Flipgrid o VideoAnt. Y, sin duda, es posible realizar un uso colaborativo vinculado a procesos de aprendizaje formal mediante redes sociales como Instagram, Twitter o herramientas como WhatsApp. De hecho, las herramientas sociales basadas en la web han demostrado ser efectivas para conectar a las personas, tanto en el nivel cognitivo como en el nivel socioemocional (Bowman y Akcaoglu, 2014; Hamid et al., 2015). Por otro lado, los estudios señalan que las tecnologías integradas en los dispositivos móviles nutren la atmósfera social, favorecen el diálogo y potencian el deseo de compartir entre los estudiantes (Bouhnik \& Deshen, 2014). En este sentido, existen evidencias de que los estudiantes prefieren utilizar medios fuera de los entornos virtuales para comunicarse $y$, además, consideran que estos mejoran los procesos de comunicación con sus grupos (Hernández, Muñoz y González, 2015).

\section{Diseño y Metodología}

La investigación se ha llevado a cabo en el contexto de los estudios universitarios de grado con la participación de estudiantes de cinco asignaturas de primer, segundo y tercer curso de dos titulaciones: Grado de Maestro en educación primaria y Grado de Maestro en educación infantil, impartidos en La Salle Campus Madrid. Las asignaturas se im- 
partieron en modalidad online y se recogió una muestra de 106 cuestionarios, el 83,46\% de la población. Con el fin de caracterizar la muestra, a continuación, se presenta la distribución de estudiantes en asignaturas y grados, así como un análisis de frecuencias y porcentajes de las variables "edad", "género" y "años de experiencia trabajando en equipo de forma colaborativa en un entorno virtual".

Tabla 1

Asignaturas participantes en el Grado en Educación infantil

\begin{tabular}{lcc}
\hline Asignatura & Curso & N Estudiantes \\
\hline Políticas educativas de la Unión Europea & $2^{\circ}$ & 23 \\
\hline Conocimiento del medio social y cultural en E.I. & $\begin{array}{c}3^{\circ} \\
\text { Grupo A }\end{array}$ & 21 \\
\hline Conocimiento del medio social y cultural en E.I. & $\begin{array}{c}3^{\circ} \\
\text { Grupo B }\end{array}$ & 2 \\
\hline
\end{tabular}

Tabla 2

Asignaturas participantes en el Grado en Educación primaria

\begin{tabular}{lcc}
\hline Asignatura & Curso & N Estudiantes \\
\hline Sociología de la Educación & $1^{\circ}$ & 12 \\
Lengua extranjera II (inglés) & $2^{\circ}$ & 30 \\
\hline
\end{tabular}

Tabla 3

Agrupación de los estudiantes participantes en rangos de edad

Frecuencia y porcentaje de la variable "edad".

\begin{tabular}{lcc}
\hline Edad & Frecuencia & Porcentaje \\
\hline Entre 21 y 25 años & 21 & 22,26 \\
Entre 26 y 30 años & 40 & 42,4 \\
Entre 31 y 35 años & 27 & 28,62 \\
Entre 36 y 40 años & 13 & 13,78 \\
Más de 40 años & 3 & 3,18 \\
No contesta & 1 & 1,06 \\
\hline
\end{tabular}

Tabla 4

Frecuencia y porcentaje de la variable "género"

\begin{tabular}{lcc}
\hline Frecuencia y porcentaje de la variable "género". & \\
\hline Género & Frecuencia & Porcentaje \\
\hline Mujer & 90 & 84,906 \\
Hombre & 16 & 15,094 \\
\hline
\end{tabular}


Herramientas que facilitan el aprendizaje colaborativo en entornos virtuales: nuevas oportunidades para el desarrollo de las ecologías digitales de aprendizaje NúRIA HeRnándEZ-SELLÉS

Tabla 5

Distribución por experiencia previa trabajando en equipo de forma colaborativa en un entorno virtual

Experiencia previa trabajando en equipo de forma colaborativa en un entorno virtual.

\begin{tabular}{lcc}
\hline & Frecuencia & Porcentaje \\
\hline Sí & 54 & 50,9 \\
No & 52 & 49,1 \\
\hline
\end{tabular}

Para garantizar unas condiciones homogéneas, se implementó un modelo de CSCL constituido por los mismos elementos e hitos en los 5 proyectos diseñados en cada asignatura, contando con el apoyo de una tutora experta que guiaba a las docentes que impartieron la docencia. El diseño se construyó sobre los siguientes elementos: 1) Instrucciones del proyecto en una guía de colaboración que incluía una descripción, justificación pedagógica del trabajo colaborativo, principales hitos relativos al desarrollo de la tarea, descripción de herramientas disponibles, propuesta para redactar unos acuerdos grupales y descripción de los valores que cimentan el trabajo colaborativo; 2) Formación espontánea de grupos por parte de los estudiantes; 3) Redacción de acuerdos grupales; 4) Revisión de los acuerdos y respuesta del docente, previa al inicio de la interacción; 5) Desarrollo de los proyectos con el apoyo de las docentes; 6) Proceso de coevaluación formativa y sumativa.

Los objetivos de investigación a los que responde el estudio son los siguientes:

- Conocer la percepción de los estudiantes en relación con la utilidad de las herramientas tecnológicas que se emplean en los contextos de CSCL

- Identificar si existen diferencias significativas en la percepción de la utilidad de las herramientas tecnológicas en función de edad de los estudiantes

- Identificar si existen diferencias significativas en la percepción de la utilidad de las herramientas tecnológicas en función de los años de experiencia trabajando en entornos virtuales

El estudio parte de una intencionalidad exploratoria y descriptiva, por ello se opta por una metodología cuantitativa de carácter no experimental de tipo encuesta (McMillan \& Schumacher, 2005). Para ello se diseñó un cuestionario con una escala tipo Likert con cinco niveles de respues- 
ta que los estudiantes respondieron de forma anónima y voluntaria. El cuestionario se estructuró en los siguientes bloques:

- BLOQUE I: Características personales y académicas.

- BLOQUE II: Organización y Gestión del trabajo colaborativo previo al desarrollo de la tarea.

- BLOQUE III: Interacción en el equipo durante el desarrollo de la tarea.

- BLOQUE IV: Evaluación y resultados de aprendizaje relacionados con el trabajo colaborativo.

- BLOQUE V: Roles y Competencias del Profesor.

- BLOQUE VI: Herramientas que soportan el trabajo colaborativo.

El orden en el que se presentan los bloques trata de responder a la secuencia de trabajo seguida por los estudiantes en las tareas cooperativas, y de este modo se espera facilitar la cumplimentación del cuestionario a los estudiantes. Así, primero se encuentran las preguntas relacionadas con el diseño del trabajo y la formación de los grupos; a continuación, se presenta la planificación de trabajo en los grupos; posteriormente, se sigue con la interacción; y se continúa con la evaluación del aprendizaje en contacto con el grupo y con unas consideraciones en torno a la evaluación.

El carácter holístico del estudio supuso un reto para la elaboración del cuestionario y la exhaustividad que requiere abordar con rigor esta globalidad resultó en un cuestionario extenso, que a pesar de ello no pareció tedioso a los estudiantes que lo cumplimentaron en la aplicación piloto con 25 de ellos. En ningún caso el tiempo de cumplimentación superó los 20 minutos. La versión final del cuestionario incorpora un total de 139 ítems distribuidos en los 6 bloques ya descritos.

Para asegurar las condiciones de validez el cuestionario se sometió a juicio de expertos y, posteriormente, se realizó un estudio piloto antes de consolidar la versión definitiva. Se solicitó la colaboración de cinco profesores universitarios del área de Didáctica y Organización Escolar (dos catedráticos y tres titulares de universidad), entre los cuales cuatro de ellos están especializados profesionalmente en tecnología educativa.

Con el fin de completar los análisis descriptivos se llevó a cabo diversos análisis estadísticos bivariados con el fin de identificar diferencias significativas en relación con la edad de los estudiantes y con los años de experiencia trabajando en entornos virtuales, aspectos recogidos en 
Herramientas que facilitan el aprendizaje colaborativo en entornos virtuales: nuevas oportunidades para el desarrollo de las ecologías digitales de aprendizaje NúRIA HeRnándEZ-SELLÉS

las preguntas iniciales dirigidas a conocer las características de los participantes. Para el contraste estadístico de variables independientes con más de dos categorías (edad y años de experiencia en entornos virtuales) se ha empleado la prueba no paramétrica Kruskal-Wallis con un riesgo de error del $5 \%(\alpha=0.05)$.

\section{Resultados}

La tabla 6 incorpora los análisis descriptivos relativos a las valoraciones de los estudiantes en relación con las herramientas que en su opinión serían útiles para trabajar en equipo en contextos de trabajo colaborativo CSCL. Se incorpora todos los ítems analizados junto con las frecuencias y porcentajes resultantes. Se incorporan asimismo las medidas de tendencia central (media) y de dispersión (desviación típica). A continuación, la figura 1 representa las puntuaciones medias de los ítems presentados en la tabla 6 .

Tabla 6

Valoración de las herramientas que serían útiles para trabajar en equipo en una experiencia similar a la vivida en la asignatura

\begin{tabular}{|c|c|c|c|c|c|c|c|c|c|c|c|c|c|c|}
\hline & \multicolumn{2}{|c|}{$\mathrm{NS} / \mathrm{NC}$} & \multicolumn{2}{|c|}{ Muy bajo } & \multicolumn{2}{|c|}{ Bajo } & \multicolumn{2}{|c|}{ Medio } & \multicolumn{2}{|c|}{ Alto } & \multicolumn{2}{|c|}{ Muy Alto } & \multirow{2}{*}{$\begin{array}{l}\text { Me- } \\
\text { dia }\end{array}$} & \multirow{2}{*}{ DT } \\
\hline & $\mathrm{n}$ & $\%$ & $n$ & $\%$ & $n$ & $\%$ & $\mathrm{n}$ & $\%$ & $n$ & $\%$ & $\mathrm{n}$ & $\%$ & & \\
\hline Wikis. & 7,0 & 6,6 & 8,0 & 7,5 & 20,0 & 18,9 & 29,0 & 27,4 & 30,0 & 28,3 & 12,0 & 11,3 & 3,18 & 1,137 \\
\hline $\begin{array}{l}\text { Redes sociales (Facebook, } \\
\text { Google +, Twitter...) }\end{array}$ & 2,0 & 1,9 & 5,0 & 4,7 & 16,0 & 15,1 & 27,0 & 25,5 & 25,0 & 23,6 & 31,0 & 29,2 & 3,59 & 1,204 \\
\hline Blogs. & 3,0 & 2,8 & 6,0 & 5,7 & 19,0 & 17,9 & 23,0 & 21,7 & 36,0 & 34,0 & 19,0 & 17,9 & 3,42 & 1,159 \\
\hline $\begin{array}{l}\text { Videoconferencia/ } \\
\text { Audioconferencia. }\end{array}$ & 4,0 & 3,8 & 9,0 & 8,5 & 14,0 & 13,2 & 29,0 & 27,4 & 20,0 & 18,9 & 30,0 & 28,3 & 3,47 & 1,287 \\
\hline $\begin{array}{l}\text { Mapas conceptuales co- } \\
\text { laborativos. }\end{array}$ & 6,0 & 5,7 & 7,0 & 6,6 & 8,0 & 7,5 & 33,0 & 31,1 & 26,0 & 24,5 & 26,0 & 24,5 & 3,56 & 1,166 \\
\hline $\begin{array}{l}\text { Mensajería instantánea } \\
\text { (Google talk, Skype...) }\end{array}$ & 2,0 & 1,9 & 1,0 & 0,9 & 2,0 & 1,9 & 10,0 & 9,4 & 25,0 & 23,6 & 66,0 & 62,3 & 4,47 & ,824 \\
\hline $\begin{array}{l}\text { Mensajería instantánea a } \\
\text { través de apps para móvi- } \\
\text { les y tablets (Whats app...) }\end{array}$ & 1,0 & 0,9 & 2,0 & 1,9 & 1,0 & 0,9 & 4,0 & 3,8 & 22,0 & 20,8 & 76,0 & 71,7 & 4,61 &, 778 \\
\hline $\begin{array}{l}\text { Marcadores sociales (Dii- } \\
\text { go, Delicious, Mr. Wong, } \\
\text { etc.) }\end{array}$ & 10 & 9,4 & 17,0 & 16,0 & 17,0 & 16,0 & 32,0 & 30,2 & 17,0 & 16,0 & 13,0 & 12,3 & 2,92 & 1,270 \\
\hline
\end{tabular}

En relación con el uso de Wikis, Redes sociales y Blogs, se observa una elevada dispersión en las respuestas, que se distribuyen con porcentajes similares entre las escalas Bajo, Medio, Alto y Muy alto. Podría inferirse 
que estas herramientas no son de uso extendido en los ámbitos educativos y cabría preguntarse si esta dispersión se debe al desconocimiento o a la falta de experiencia en las prácticas de enseñanza-aprendizaje.

En cuanto a las videoconferencias, las respuestas se concentran en los ítems Medio (27,4\%), Alto (18,9\%) y Muy Alto (28,3\%), sumando un $74,6 \%$. Cabría preguntarse, a pesar de este dato, y dado el contexto actual, en el que se han multiplicado las prácticas educativas a través de videoconferencias, a qué se debe que un porcentaje no desdeñable de estudiantes, las valore poco positivamente.

Respecto al uso de mapas conceptuales las respuestas se concentran en las categorías Medio (31,1\%), Alto (24,5\%) y Muy Alto (24,5\%). Se infiere que las herramientas de representación les parecen de utilidad.

Lo que es rotundo es que, tanto la Mensajería instantánea (Google talk, Skype...), como la Mensajería instantánea a través de Apps para móviles y tablets, tipo WhatsApp, son las herramientas más valoradas por los estudiantes, obteniendo una valoración más alta con medias de 4,47 y 4,61, respectivamente. Por otro lado, cabe destacar que los resultados se encuentran concentrados en las categorías: Alto y Muy Alto.

Por último, en relación con los Marcadores sociales (Diigo, Delicious, Mr. Wong, etc.) se observa una elevada dispersión en las respuestas, que se distribuyen con porcentajes similares entre las escalas Bajo, Medio, Alto y Muy alto. La media de 2,92 es la más baja del bloque.

En cuanto a las pruebas estadísticas no paramétricas, se ha trabajado el contraste de medias con el objetivo de identificar diferencias significativas entre las variables analizadas. En las tablas 7 y 8 se presentan los resultados del contraste según la variable edad y la variable años de experiencia utilizando sistemas de aprendizaje colaborativo. El rango de medias es el promedio de los rangos de todas las observaciones de cada muestra. Cuando el rango de medias de un grupo es mayor que el rango promedio general, los valores de las observaciones de ese grupo tienden a ser mayores que los de los otros grupos. 
Herramientas que facilitan el aprendizaje colaborativo en entornos virtuales: nuevas oportunidades para el desarrollo de las ecologías digitales de aprendizaje

NúRIA HeRnándEZ-SELLÉS

Tabla 7

Estadísticos de contraste edad* herramientas que serían útiles para trabajar en equipo en una experiencia similar a la vivida en la asignatura

\begin{tabular}{|c|c|c|c|c|}
\hline & edad & $\mathrm{N}$ & $\begin{array}{l}\text { Rango } \\
\text { promedio }\end{array}$ & $\begin{array}{l}\text { Estadísticos de } \\
\text { contraste }\end{array}$ \\
\hline \multirow{4}{*}{ Wikis. } & Entre 20 y 24 años & 12 & 44,58 & \multirow{4}{*}{$\begin{array}{l}\text { Chi-Cuadrado: ,581 } \\
\text { Gl: } 2 \\
\text { Sig. Asintót.: ,748 }\end{array}$} \\
\hline & Entre 25 y 29 años & 32 & 48,72 & \\
\hline & Más de 29 años & 54 & 51,06 & \\
\hline & Total & 98 & & \\
\hline \multirow{4}{*}{$\begin{array}{l}\text { Redes sociales } \\
\text { (Facebook, Tuenti, } \\
\text { Google +, Twitter...) }\end{array}$} & Entre 20 y 24 años & 15 & 63,97 & \multirow{4}{*}{$\begin{array}{l}\text { Chi-Cuadrado: 3,062 } \\
\text { Gl: } 2 \\
\text { Sig. Asintót.: ,216 }\end{array}$} \\
\hline & Entre 25 y 29 años & 33 & 50,94 & \\
\hline & Más de 29 años & 55 & 49,37 & \\
\hline & Total & 103 & & \\
\hline \multirow{4}{*}{ Blogs. } & Entre 20 y 24 años & 14 & 49,21 & \multirow{4}{*}{$\begin{array}{l}\text { Chi-Cuadrado: ,492 } \\
\text { Gl: } 2 \\
\text { Sig. Asintót.: ,782 }\end{array}$} \\
\hline & Entre 25 y 29 años & 33 & 49,41 & \\
\hline & Más de 29 años & 55 & 53,34 & \\
\hline & Total & 102 & & \\
\hline \multirow{4}{*}{$\begin{array}{l}\text { Videoconferencia/ } \\
\text { Audioconferencia. }\end{array}$} & Entre 20 y 24 años & 15 & 43,80 & \multirow{4}{*}{$\begin{array}{l}\text { Chi-Cuadrado: 6,348 } \\
\text { Gl: } 2 \\
\text { Sig. Asintót.: ,042 }\end{array}$} \\
\hline & Entre 25 y 29 años & 31 & 42,94 & \\
\hline & Más de 29 años & 55 & 57,51 & \\
\hline & Total & 101 & & \\
\hline \multirow{4}{*}{$\begin{array}{l}\text { Mapas conceptua- } \\
\text { les colaborativos. }\end{array}$} & Entre 20 y 24 años & 13 & 35,35 & \multirow{4}{*}{$\begin{array}{l}\text { Chi-Cuadrado: 5,516 } \\
\text {-Gl: } 2 \\
\text {-Sig. Asintót.: ,063 }\end{array}$} \\
\hline & Entre 25 y 29 años & 32 & 47,77 & \\
\hline & Más de 29 años & 54 & 54,85 & \\
\hline & Total & 99 & & \\
\hline \multirow{4}{*}{$\begin{array}{l}\text { Mensajería instan- } \\
\text { tánea (Google talk, } \\
\text { Messenger, Sky- } \\
\text { pe...) }\end{array}$} & Entre 20 y 24 años & 15 & 42,70 & \multirow{4}{*}{$\begin{array}{l}\text { Chi-Cuadrado: 4,365 } \\
\text { Gl: } 2 \\
\text { Sig. Asintót.: ,113 }\end{array}$} \\
\hline & Entre 25 y 29 años & 32 & 48,50 & \\
\hline & Más de 29 años & 56 & 56,49 & \\
\hline & Total & 103 & & \\
\hline \multirow{4}{*}{$\begin{array}{l}\text { Mensajería instantá- } \\
\text { nea a través de apps } \\
\text { para móviles y ta- } \\
\text { blets (Whats app...) }\end{array}$} & Entre 20 y 24 años & 15 & 55,97 & \multirow{4}{*}{$\begin{array}{l}\text { Chi-Cuadrado: 6,071 } \\
\text { Gl: } 2 \\
\text { Sig. Asintót.: ,048 }\end{array}$} \\
\hline & Entre 25 y 29 años & 33 & 44,21 & \\
\hline & Más de 29 años & 56 & 56,46 & \\
\hline & Total & 104 & & \\
\hline \multirow{4}{*}{$\begin{array}{l}\text { Marcadores sociales } \\
\text { (Diigo, Delicious, } \\
\text { Mr. Wong, etc.) }\end{array}$} & Entre 20 y 24 años & 13 & 52,85 & \multirow{4}{*}{$\begin{array}{l}\text { Chi-Cuadrado: 2,187 } \\
\text { Gl: } 2 \\
\text { Sig. Asintót.: ,335 }\end{array}$} \\
\hline & Entre 25 y 29 años & 32 & 42,42 & \\
\hline & Más de 29 años & 50 & 50,31 & \\
\hline & Total & 95 & & \\
\hline
\end{tabular}

Los contrastes estadísticos nos señalan que, en función de la edad de los estudiantes existen diferencias significativas en la percepción de utilidad de dos tipos de herramientas, en concreto: Videoconferencia/ Au- 
dioconferencia y Mensajería instantánea a través de Apps para móviles y tablets (Whats app...). Los estudiantes con más de 29 años, en ambos casos, muestran preferencia por la videoconferencia/audioconferencia y por las herramientas basadas en la mensajería instantánea utilizadas desde dispositivos móviles.

Tabla 8

Estadísticos de contraste años de experiencia en entornos virtuales* herramientas que serían útiles para trabajar en equipo en una experiencia similar a la vivida en la asignatura

\begin{tabular}{|c|c|c|c|}
\hline & $\begin{array}{l}\text { años de experien- } \\
\text { cia en entornos } \\
\text { virtuales }\end{array}$ & $\mathrm{N}$ & $\begin{array}{l}\text { Rango Estadísticos de } \\
\text { prome- } \\
\text { dio contraste }\end{array}$ \\
\hline \multirow{4}{*}{ Wikis. } & Años_ninguno & 30 & \multirow{4}{*}{$\begin{array}{l}\text {-Chi-Cuadrado: 1,681 } \\
\text {-Gl: } 2 \\
\text {-Sig. Asintót.: ,432 }\end{array}$} \\
\hline & Años_hasta1 & 30 & \\
\hline & Años_mas1 & 38 & \\
\hline & Total & 98 & \\
\hline \multirow{4}{*}{$\begin{array}{l}\text { Redes sociales (Face- } \\
\text { book, Tuenti, Google +, } \\
\text { Twitter...) }\end{array}$} & Años_ninguno & 32 & \multirow{4}{*}{$\begin{array}{l}\text {-Chi-Cuadrado: 11,466 } \\
\text {-Gl: } 2 \\
\text {-Sig. Asintót.: ,003 }\end{array}$} \\
\hline & Años_hasta1 & 32 & \\
\hline & Años_mas1 & 39 & \\
\hline & Total & 103 & \\
\hline \multirow{4}{*}{ Blogs. } & Años_ninguno & 32 & \multirow{4}{*}{$\begin{array}{l}\text {-Chi-Cuadrado: 1,207 } \\
\text {-Gl: } 2 \\
\text {-Sig. Asintót.: ,547 }\end{array}$} \\
\hline & Años_hasta1 & 31 & \\
\hline & Años_mas1 & 39 & \\
\hline & Total & 102 & \\
\hline \multirow{4}{*}{$\begin{array}{l}\text { Videoconferencia/ Au- } \\
\text { dioconferencia. }\end{array}$} & Años_ninguno & 31 & \multirow{4}{*}{$\begin{array}{l}\text {-Chi-Cuadrado: ,592 } \\
\text {-Gl: } 2 \\
\text {-Sig. Asintót.: ,744 }\end{array}$} \\
\hline & Años_hasta1 & 31 & \\
\hline & Años_mas1 & 39 & \\
\hline & Total & 101 & \\
\hline \multirow{4}{*}{$\begin{array}{l}\text { Mapas conceptuales } \\
\text { colaborativos. }\end{array}$} & Años_ninguno & 32 & \multirow{4}{*}{$\begin{array}{l}\text {-Chi-Cuadrado: 5,848 } \\
\text {-Gl: } 2 \\
\text {-Sig. Asintót.: ,054 }\end{array}$} \\
\hline & Años_hasta1 & 29 & \\
\hline & Años_mas1 & 38 & \\
\hline & Total & 99 & \\
\hline \multirow{4}{*}{$\begin{array}{l}\text { Mensajería instantánea } \\
\text { (Google talk, Messen- } \\
\text { ger, Skype...) }\end{array}$} & Años_ninguno & 32 & \multirow{4}{*}{$\begin{array}{l}\text {-Chi-Cuadrado: 1,901 } \\
\text {-Gl: } 2 \\
\text {-Sig. Asintót.: ,387 }\end{array}$} \\
\hline & Años_hasta1 & 33 & \\
\hline & Años_mas1 & 38 & \\
\hline & Total & 103 & \\
\hline \multirow{4}{*}{$\begin{array}{l}\text { Mensajería instantá- } \\
\text { nea a través de apps } \\
\text { para móviles y tablets } \\
\text { (Whats app...) }\end{array}$} & Años_ninguno & 32 & \multirow{4}{*}{$\begin{array}{l}\text {-Chi-Cuadrado: 2,586 } \\
\text {-Gl: } 2 \\
\text {-Sig. Asintót.: ,274 }\end{array}$} \\
\hline & Años_hasta1 & 33 & \\
\hline & Años_mas1 & 39 & \\
\hline & Total & 104 & \\
\hline
\end{tabular}




\begin{tabular}{|c|c|c|c|c|}
\hline & $\begin{array}{l}\text { años de experien- } \\
\text { cia en entornos } \\
\text { virtuales }\end{array}$ & $\mathrm{N}$ & $\begin{array}{l}\text { Rango } \\
\text { prome- } \\
\text { dio }\end{array}$ & $\begin{array}{l}\text { Estadísticos de } \\
\text { contraste }\end{array}$ \\
\hline \multirow{4}{*}{$\begin{array}{l}\text { Marcadores sociales } \\
\text { (Diigo, Delicious, Mr. } \\
\text { Wong, etc.) }\end{array}$} & Años_ninguno & 32 & 51,22 & \multirow{4}{*}{$\begin{array}{l}\text {-Chi-Cuadrado: ,722 } \\
\text {-Gl: } 2 \\
\text {-Sig. Asintót.: ,697 }\end{array}$} \\
\hline & Años_hasta1 & 29 & 46,93 & \\
\hline & Años_mas1 & 34 & 45,88 & \\
\hline & Total & 95 & & \\
\hline
\end{tabular}

Los contrastes estadísticos nos señalan que, en función de los años de experiencia en entornos virtuales existen diferencias significativas en la percepción de utilidad de "Redes sociales (Facebook, Google +, Twitter...)". En este caso, los estudiantes con menos años de experiencia trabajando en entornos virtuales, en concreto, aquellos con ningún año de experiencia, son los que consideran que las redes sociales pueden ser de mayor utilidad para apoyar procesos de CSCL.

\section{Discusión y conclusiones}

Las respuestas de los estudiantes en relación con el uso de herramientas como wikis, redes sociales y blogs, al igual que en relación con el aprendizaje vinculado con el uso de marcadores sociales, nos habla de la necesidad de seguir investigando y aplicando su empleo en los contextos educativos, de manera que se puedan generar nuevas oportunidades. Esto resulta especialmente relevante por cuanto estas herramientas del entorno de la Web Social son vehículos accesibles y amigables para propiciar e impulsar la aparición de un mayor número de intercambios de diversa índole, no solo relacionados con el ámbito cognitivo, sino también con el emocional (Hamid et al., 2015). Es señalable que los estudiantes que mejor han valorado las redes sociales son aquellos con menos años de experiencia trabajando en entornos de aprendizaje virtuales, pero que sí han tenido acceso a estas herramientas para un uso social o personal.

Un aspecto destacable es que los estudiantes están en su mayoría satisfechos con el sistema de videoconferencia en el contexto de la formación virtual y, en concreto, en el desarrollo de CSCL, e particular los mayores de 29 años. Sin embargo, será necesario continuar indagando para averiguar qué sucede con aquellos que no se sienten satisfechos uti- 
lizando las videoconferencias; cuáles son sus dificultades y los aspectos que necesitan ser mejorados.

Otro aspecto de interés es que los estudiantes han valorado positivamente el uso de herramientas visuales como los mapas conceptuales. Esto evidencia el gran reto existente en las instituciones de educación superior para integrar la cultura de consumo multimedia que los estudiantes realizan, sobre todo, a través de sus dispositivos móviles en los procesos de enseñanza-aprendizaje, como parte de su compromiso de alinearse con los cambios sociales y culturales.

Por otro lado, una cuestión de gran importancia es el hecho de que los estudiantes y en particular, aquellos mayores de 29 años, muestren una preferencia por utilizar herramientas de mensajería instantánea a través de dispositivos móviles tipo WhatsAPP o bien otras aplicaciones como Skype. Parece claro que estas herramientas cotidianas que están integradas en el proceso de comunicación de nuestros estudiantes se consideran de gran valor para sostener el trabajo colaborativo. Los resultados de este estudio ponen en evidencia que los estudiantes universitarios nos están indicando que desearían utilizarlas en el ámbito formal y debemos seguir profundizando en las fórmulas que faciliten esa integración.

En este sentido, será importante indagar tanto en las preferencias de los estudiantes como en los modos de consumo que ellos desarrollan en los medios o herramientas, ya que no solo es necesario seleccionar el medio sino también revisar la forma en la que ellos emplean las herramientas a nivel personal, en su ocio o sus relaciones. Así se podrían aprovechar esos conocimientos y habilidades del alumnado para transferirlos al ámbito educativo y adaptar las aplicaciones digitales a los contextos de enseñanza. Existen experiencias que demuestran la eficacia de estos medios en los ámbitos formales de aprendizaje (Bouhnik \& Deshen, 2014) y que evidencian un dilema entre el uso de las plataformas tradicionales y aquellas herramientas sociales caracterizadas por la gratuidad, rapidez y conexión desde dispositivos móviles, que son más accesibles y facilitan la continuidad de los contactos (Hernández, Muñoz y González, 2014b). Sin embargo, también es necesario contemplar que los grandes flujos de información y la necesidad de inmediatez pueden provocar que los estudiantes reclamen una atención continuada e inmediata por parte de los profesores, lo cual puede generar ansiedad en los docentes (Hernández et al., 2014b).

En las instituciones educativas, cualquier apuesta tecnológica que 
Herramientas que facilitan el aprendizaje colaborativo en entornos virtuales: nuevas oportunidades para el desarrollo de las ecologías digitales de aprendizaje Núria HeRnández-SeLlés

sustente los aprendizajes necesita pasar por una revisión estratégica que, tomando como base la investigación y las experiencias de aula presentes en su institución y también en otras organizaciones de referencia, pueda promover una cultura con sentido para la comunidad. Este es un buen momento para recoger las experiencias forzadas por la situación de pandemia cara a promover una continuidad de las mejores prácticas y experiencias y, sobre todo, para aprender de aquellas que no han dado los frutos esperados. Es una oportunidad para dar sentido y respaldo al gran esfuerzo que han realizado los claustros y los estudiantes, que, a pesar de las dificultades, han podido constatar la utilidad de la tecnología como mediadora de los aprendizajes iniciando un camino que continuará y se ampliará irremediablemente. De ahí la necesidad de contar con investigaciones que contribuyan a fundamentar las decisiones que han de tomar los responsables de las instituciones de educación superior en favor de la tecnología como recurso facilitador de los aprendizajes. Pero, además, teniendo en cuenta la relevancia de la tecnología como un componente fundamental de las ecologías de aprendizaje digital (González-Sanmamed, Muñoz-Carril y Santos-Caamaño, 2019; González-Sanmamed, Sangrà, Souto-Seijo y Estevez, 2020; Romeu-Fontanillas, Guitert-Catasús, Raffaghelli y Sangrà, 2020 , tanto alumnado como profesorado, tendrán que asumir la imperiosa necesidad del desarrollo de sus competencias digitales como uno de los requisitos que contribuirá a facilitar sus aprendizajes a lo largo y ancho de su vida personal y profesional (Estévez, Souto-Seijo, Sande y González-Sanmamed, 2020; Matzat y Vrieling, 2016; Peters y Romero, 2019; Souto-Seijo, Estévez, Iglesias y González-Sanmamed, 2020; Souto-Seijo, Estévez, Romero y González-Sanmamed, 2020).

\section{Limitaciones del estudio}

Este estudio tiene limitaciones potenciales. Sería interesante incrementar el número de participantes, así como su grado de heterogeneidad, por ejemplo, con estudiantes de distintas universidades que ofrecen educación online o mediada por tecnologías que impartan docencia utilizando CSCL en las asignaturas. Además, sería interesante incrementar la heterogeneidad teniendo en cuenta titulaciones de diferentes áreas de conocimiento. 
Herramientas que facilitan el aprendizaje colaborativo en entornos virtuales: nuevas oportunidades para el desarrollo de las ecologías digitales de aprendizaje

NúrIA HeRnández-Sellés

\section{Referencias}

Bouhnik, D., y Deshen, M. (2014). WhatsApp goes to school: Mobile instant messaging between teachers and students. Journal of Information Technology Education: Research, 13, 217-231. doi 10.28945/2051

Abdu, R., DeGroot, R., y Drachman, R., (2012). Teacher's role in computer supported collaborative learning. Proceedings of the 7th Chais Conference for Innovation in Learning Technologies, 1-6. Raanana: The Open University of Israel.

Bowman, N. D., y Akcaoglu, M. (2014). "I see smart people!": Using Facebook to supplement cognitive and affective learning in the university mass lecture. Internet and Higher Education, 23, 1-8. doi:10.1016/j.iheduc.2014.05.003

Darling-Hammond, L. y Hyler, M. (2020). Preparing educators for the time of COVID... and beyond. European Journal of Teacher Education. doi:10.1080/02619768.2020. 1816961

Estévez, I., Souto-Seijo, A., Sande, O., y González-Sanmamed, M. (2020). Aprendizaje Profesional Docente a través de la Autoformación: un Análisis Cualitativo de las Ecologías de Aprendizaje. En S. Oliveira e Sá, F. Freitas, P. Castro, M. González-Sanmamed, y A. P. Costa (Eds.), Investigación Cualitativa en Educación: avances y desafíos (Vol. 2, pp. 437-447). Doi:10.36367/ntqr.2.2020.437-447

Garrison, D. R., y Cleveland-Innes, M. (2005). Facilitating cognitive presence in online learning: interaction is not enough. American Journal of Distance Education, 19(3), 133-148. doi:10.1207/s15389286ajde1903_2

Garrison, D. R., Cleveland-Innes, M., y Fung, T. S. (2010). Exploring causal relationships among teaching, cognitive and social presence: Student perceptions of the community of inquiry framework. Internet and Higher Education, 13(1), 31-36. doi:10.1016/j. iheduc.2009.10.002

González-Sanmamed, M.; Muñoz-Carril, P.C. y Santos, F. (2019). Key components of learning ecologies: a Delphi assessment. British Journal of Educational Technology. 50(4), 1639-1655. doi:10.1111/bjet.12805

González-Sanmamed, M., Sangrà, A., Souto-Seijo, A., y Estévez, I. (2020). Learning ecologies in the digital era: challenges for higher education. Publicaciones, 50(1), 83-102. doi: https://doi.org/10.30827/publicaciones.v50i1.15671.

Hamid, S., Waycott, J., Kurnia, S., y Chang, S. (2015). Understanding students' perceptions of the benefits of online social networking use for teaching and learning. Internet and Higher Education, 26, 1-9. doi:10.1016/j.iheduc.2015.02.004

Hernández, N., González, M., y Muñoz, P. C. (2014a). Planning collaborative learning in virtual environments. Comunicar, 42, 25-33. doi:10.3916/C42-2014-02.

Hernández, N., Muñoz, P.C. y González, M. (2014b). CSCL: El papel de las herramientas tecnológicas como soporte del trabajo colaborativo en entornos en línea. En Strategies for edutacion in a new context, International Conference on Innovation, Documentation and Teaching Technologies. UPV (Universidad Politécnica de Valencia).

Hernández, N., Muñoz, P.C. y González, M. (2015) Selección de las tecnologías que facilitan el aprendizaje colaborativo en entornos virtuales". Congreso Virtual Iberoamericano sobre Recursos Educativos Innovadores. Universidade Aberta, Universidad de

https://doi.org/10.6018/educatio.465741 
Herramientas que facilitan el aprendizaje colaborativo en entornos virtuales: nuevas oportunidades para el desarrollo de las ecologías digitales de aprendizaje

NúRIA HeRnándEZ-SELLÉS

Alcalá, Universidad Federal do Rio Grande do Sul.

Hernández, N., Muñoz, P.C. y González, M. (2018). La e-evaluación en el trabajo colaborativo en entornos virtuales: Análisis de la percepción de los estudiantes. Edutec-E, 65, 16-28. doi:10.21556/edutec.2018.65.997

Hernández, N., Muñoz, P.C. y González, M. (2019). Computer-supported collaborative learning: An analysis of the relationship between interaction, emotional support and online collaborative tools. Computers \& Education, 138, 1-12. DOI: doi:1016/j.compedu.2019.04.012

Johnson, D. W., Johnson, R. T., y Stanne, M. B. (2000). Cooperative learning methods: A meta-analysis. Minneapolis, MN: University of Minnesota.

Johnson, D. W., y Johnson, R. T. (2004). Cooperation and the use of technology. En D. $\mathrm{H}$. Johanssen. (2nd ed.), Handbook of research on educational communications and technology, 785-811. Mahwah, NJ: Lawrence Erlbaum Associates.

King, A. (2007). Scripting collaborative learning processes: a cognitive perspective. In Fischer, F., Kollar, I., Mandl, H., Haake, J.M. (Eds.), Scripting computer-supported collaborative learning: Cognitive, computational and educational perspectives (pp. 13-37). New York: Springer. doi:10.1007/978-0-387-36949-5_2

Kwon, K., Liu, Y., y Johnson, L. (2014). Group regulation and social-emotional interactions observed in computer supported collaborative Learning: Comparison between good vs. poor collaborators. Computers \& Education, 78, 185-200. doi:10.1016/j. compedu.2014.06.004

Lu, J., Lajoie, S.P., y Wiseman, J. (2010) Scaffolding Problem-Based Learning with CSCL Tools in International Journal of Computer-Supported Collaborative Learning, 5(3), 283-298. doi:10.1007/s11412-010-9092-6

Matzat, U. \& Vrieling, E.M. (2016). Self-regulated learning and social media -a 'natural alliance'? Evidence on students' self-regulation of learning, social media use, and student-teacher relationship. Learning, Media and Technology, 41(1), 73-99. https:// doi.org/10.1080/17439884.2015.1064953

McMillan, J., y Schumacher, S. (2010). Research in Education: Evidence-Based Inquiry. Harlow: Pearson Addison Wesley.

Medina, R., y Suthers, D. (2008). Bringing representational practice from log to light. Proceedings of the 8th international conference on International conference for the learning sciences, 59-66.

Oakley, B. Felder, R.M., Brent, R., y Elhajj, I. (2004). Turning Student Groups into Effective Teams. Journal of Student Centered Learning, 2(1), 9-34.

Onrubia, J., y Engel, A. (2012). The role of teacher assistance on the effects of a macroscript in collaborative writing tasks. International Journal of Computer-Supported CoIlaborative Learning, 7(1), 161-186. doi:10.1007/s11412-011-9125-9

Pachler, N., Daly, C., Mor, Y., y Mellar, H. (2010). Formative e-assessment: practitioner cases. Computers \& Education, 54(3), 715-21. doi:10.1016/j.compedu.2009.09.032

Peters, M. \& Romero, M. (2019). Lifelong learning ecologies in online higher education: Students' engagement in the continuum between formal and informal learning. British Journal of Educational Technology, 50(4), 1729-1743. https://doi.org/10.1111/ bjet.12803 
Herramientas que facilitan el aprendizaje colaborativo en entornos virtuales: nuevas oportunidades para el desarrollo de las ecologías digitales de aprendizaje

NúrIA HeRnández-Sellés

Reyes, M. R., Brackett, M. A., Rivers, S. E., White, M., y Salovey, P. (2012). Classroom emotional climate, student engagement, and academic achievement. Journal of Educational Psychology, 104, 700-712. doi:10.1037/a0027268

Romeu-Fontanillas, T., Guitert, M., Raffaghelli, J.-E., y Sangrà, A. (2020). Ecologías de aprendizaje para usar las TIC inspirándose en docentes referentes. Comunicar, 28(62). doi: 10.3916/c62-2020-03

Scardamalia, M., y Bereiter, C. (1994). Computer support for knowledge-building communities. The Journal of the Learning Sciences, 3(3), 265-283. doi:10.1207/ s15327809jls0303_3

Souto-Seijo, A., Estévez, I., Iglesias, V., y González-Sanmamed, M. (2020). Entre lo formal y lo no formal: un análisis desde la formación permanente del profesorado. Educar 56(1), 97-107. doi: 10.5565/rev/educar.1095

Souto-Seijo, A., Estévez, I., Romero, P., y González-Sanmamed, M. (2020). Aprendizajes Formales, No Formales e Informales en la Era Digital: Contribuciones al Desarrollo Profesional Docente. En S. Oliveira e Sá, F. Freitas, P. Castro, M. González-Sanmamed, y A. P. Costa (Eds.), Investigación Cualitativa en Educación: avances y desafíos (Vol. 2, pp. 428-436). En https://doi.org/10.36367/ntqr.2.2020.428-436

Strijbos, J., Martens, R., y Jochems, W. (2004). Designing for Interaction: Six Steps to Designing Computer-Supported Group-based Learning. Computers \& Education, 42, 403-424. doi:10.1016/j.compedu.2003.10.004

Tang, KY., Wang, CY., Chang, HY. et al. The Intellectual Structure of Metacognitive Scaffolding in Science Education: A Co-citation Network Analysis. Int J of Sci and Math Educ 14, 249-262 (2016). doi:10.1007/s10763-015-9696-4

\section{Agradecimientos}

Este trabajo se ha elaborado en el marco del proyecto de investigación titulado: "Ecologías de aprendizaje en la era digital: nuevas oportunidades para la formación del profesorado de educación secundaria" (ECO4 LEARN-SE), parcialmente financiado por el Ministerio de Ciencia, Innovación y Universidades (Referencia RTI2018-095690-B-I00). 
